\title{
Management of acute coronary syndrome-renal outcomes
}

Early invasive management of acute coronary syndrome (that is, coronary angiography with or without percutaneous coronary intervention within $48 \mathrm{~h}$ of hospital admission) is associated with improved patient survival without an increase in the risk of dialysis or of end-stage renal disease (ESRD), say researchers.

"A key element of quality care for patients with heart disease is access to cardiac procedures when necessary," says study author Matthew James. "Kidney disease is one of the strongest risk factors for heart disease but very little data on the risks and benefits of cardiac procedures in patients with kidney disease are available." To address this problem, James et al. analysed renal outcomes and mortality in 10,516 adults with non-ST elevation acute coronary syndrome who were admitted to Canadian hospitals between January 2004 and October 2009 and underwent an assessment of kidney function on admission.

The researchers report that $40.7 \%$ of the study participants were managed using an early invasive approach. Patients with lower estimated glomerular filtration rates (eGFRs) at baseline were more likely to receive conservative management. In their propensity-score-matched analysis, the researchers found that patients who received invasive management had a higher risk of acute kidney injury (AKI) - but not of AKI requiring dialysisthan did patients who received conservative management. They observed no significant

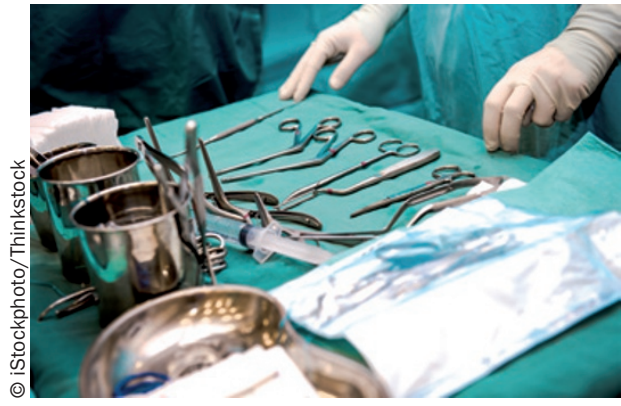

differences between the treatment groups in the risks of reinfarction, congestive heart failure, stroke, blood transfusion or death during the index hospital admission or in the risk of ESRD during a median follow-up of 2.5 years. However, early invasive management was associated with significantly better patient survival than was conservative management. The researchers report that the relative risks of AKI, ESRD and mortality associated with early invasive versus conservative management did not differ according to baseline eGFR.

"Our study suggests that the lifesaving benefits of angioplasty and bypass surgery for patients with acute coronary syndromes may extend to those who also have kidney disease," concludes James. "This information will help patients with kidney disease and their doctors to decide on the best treatment options for acute coronary syndromes."

Ellen F. Carney

Original article James, M. T. et al. Renal outcomes associated with invasive versus conservative management of acute coronary syndrome: propensity matched cohort study. BMJ doi:10.1136/bmj.f4151 